\title{
Micro-mutual-dipolar model for rapid calculation of forces between paramagnetic colloids
}

\author{
Di Du and Sibani Lisa Biswal* \\ Department of Chemical and Biomolecular Engineering, Rice University, Houston, Texas 77005, USA
}

(Received 13 June 2014; published 24 September 2014)

\begin{abstract}
Typically, the force between paramagnetic particles in a uniform magnetic field is calculated using either dipole-based models or the Maxwell stress tensor combined with Laplace's equation for magnetostatics. Dipolebased models are fast but involve many assumptions, leading to inaccuracies in determining forces for clusters of particles. The Maxwell stress tensor yields an exact force calculation, but solving Laplace's equation is very time consuming. Here, we present a more elaborate dipole-based model: the micro-mutual-dipolar model. Our model has a time complexity that is similar to that of other dipole-based models but is much more accurate especially when used to calculate the force of small aggregates. Using this model, we calculate the force between two paramagnetic spheres in a uniform magnetic field and a circular rotational magnetic field and compare our results with those of other models. The forces for three-particle and ten-particle systems dispersed in two-dimensional (2D) space are examined using the same model. We also apply this model to calculate the force between two paramagnetic disks dispersed in 2D space. The micro-mutual-dipolar model is demonstrated to be useful for force calculations in dynamic simulations of small clusters of particles for which both accuracy and efficiency are desirable.
\end{abstract}

DOI: 10.1103/PhysRevE.90.033310

PACS number(s): 02.60.Lj, 82.70.-y, 36.40.Cg, 41.20.Gz

Paramagnetic colloidal particles suspended in liquid form different structures, such as chains [1], sheets [2], membranes [3], and networks [4], in the presence of different types of magnetic fields. The versatility of structure manipulation enables paramagnetic colloidal suspensions to have numerous applications, such as smart fluids [5,6], biomedical sensing $[7,8]$, propulsion and transportation in fluids $[9,10]$, and force probes [11,12]. In these applications, the magnetic force between paramagnetic particles must be calculated accurately. Typically, dipole-based models, such as the dipolar model (DM) and the mutual-dipolar model (MDM), are used to calculate the force between paramagnetic particles placed in an external magnetic field [2,5,13]. Dipole-based models are usually fast but inaccurate for systems in which particles are close to one another. Such models are inaccurate because they do not consider multipolar effects [14]. The exact force can be calculated by solving a Laplace equation for magnetostatics with multiple boundary and initial conditions and calculating the Maxwell stress tensor for each particle [15]. The solution to the Laplace equation can be analytically approximated by a solid harmonics expansion with the Hobson formula applied to unify the coordinate system $[14,16]$. This coordinate unification is very computationally expensive and suffers from singularity-related issues $[17,18]$. A numerical solution to Laplace's equation can be obtained by using a smoothed representation of susceptibility to replace the boundary conditions [17]; this method is referred to as the Laplace equation solver (LES) method. This numerical approach is stable in terms of error propagation but still computationally time consuming.

Here, we present a more-sophisticated dipole-based model that considers mutual interactions between dipole moments and multipolar effects. All dipole-based models are based on the fact that a single spherical paramagnetic particle is uniformly magnetized in the presence of a uniform magnetic

*Corresponding author: biswal@ rice.edu field [13]. In three-dimensional (3D) space, such a sphere acquires a magnetic dipole moment $\mathbf{m}=\frac{4 \pi a^{3} \chi_{\text {eff }} \mathbf{H}_{0}}{3}$, where $a$ is the sphere's radius, $\chi_{\text {eff }}=\frac{3 \chi}{3+\chi}$ is the effective volumetric susceptibility of the sphere, $\chi$ is the magnetic susceptibility of the material, and $\mathbf{H}_{\mathbf{0}}$ is the applied magnetic field. When two identical spheres are placed a distance $r$ away from each other, if the uniform magnetization is assumed to be maintained, the force between the two spheres can be approximated as [13]

$$
\begin{aligned}
\mathbf{F}= & \frac{3 \mu_{0}}{4 \pi r^{5}}\left[\left(\mathbf{m}_{1} \cdot \mathbf{r}\right) \mathbf{m}_{2}+\left(\mathbf{m}_{2} \cdot \mathbf{r}\right) \mathbf{m}_{1}+\left(\mathbf{m}_{1} \cdot \mathbf{m}_{2}\right) \mathbf{r}\right. \\
& \left.-\frac{5\left(\mathbf{m}_{1} \cdot \mathbf{r}\right)\left(\mathbf{m}_{2} \cdot \mathbf{r}\right)}{r^{2}} \mathbf{r}\right]
\end{aligned}
$$

where $\mathbf{F}$ is the force exerted on sphere $1, \mathbf{m}_{1}=\frac{4 \pi a^{3} \chi_{\text {eff }} \mathbf{H}_{0}}{3}$ is the dipole moment of sphere $1, \mathbf{m}_{2}=\frac{4 \pi a^{3} \chi_{\text {eff }} \mathbf{H}_{0}}{3}$ is the dipole moment of sphere $2, \mu_{0}$ is the vacuum permeability, and $\mathbf{r}$ is the connector vector from sphere 1 to sphere 2, which has magnitude $r$. The DM is inaccurate in two aspects: (1) The dipoles mutually interact with the dipole-induced magnetic field, and (2) the induced magnetic field distorts the uniform magnetization of the sphere, thereby generating higher-order multipolar fields rather than the simple superposition of dipolar fields. The MDM accounts for mutual interactions by modifying the dipole moment expression as follows:

$$
\mathbf{m}_{n}=\frac{4}{3} \pi a^{3} \chi_{\mathrm{eff}}\left(\mathbf{H}_{\mathbf{0}}+\sum_{k=1, k \neq n}^{N} \mathbf{H}_{\mathrm{dip}}\left(\mathbf{r}_{n}-\mathbf{r}_{k}\right)\right),
$$

where $\mathbf{H}_{\mathrm{dip}}(\mathbf{r})=\frac{\mathbf{1}}{\mathbf{4} \pi}\left(\frac{3 \mathbf{r}(\mathbf{m} \cdot \mathbf{r})}{\mathbf{r}^{5}}-\frac{\mathbf{m}}{\mathbf{r}^{3}}\right)$ is the dipole-induced magnetic field, $N$ is the number of spheres, and $\mathbf{r}_{n}$ 's are the positions of the dipole moments for $n=1,2, \ldots, N$. Nevertheless the MDM's neglect of multipolar effects leads to a significant deviation in the calculated force in the near field [17]. The multipolar effects are caused by the asymmetric magnetization inside the spheres when they are placed close 
(a)

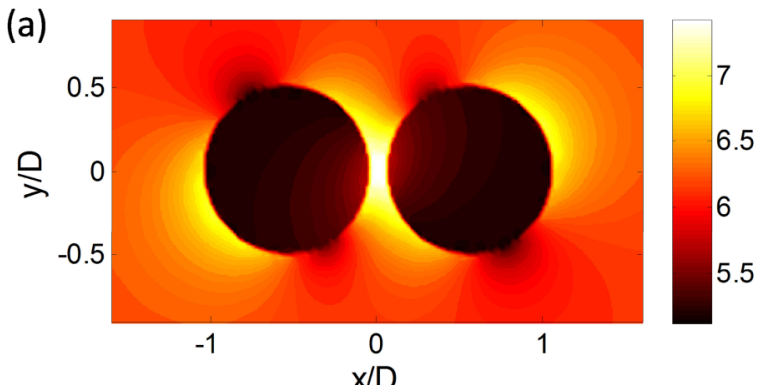

(b)
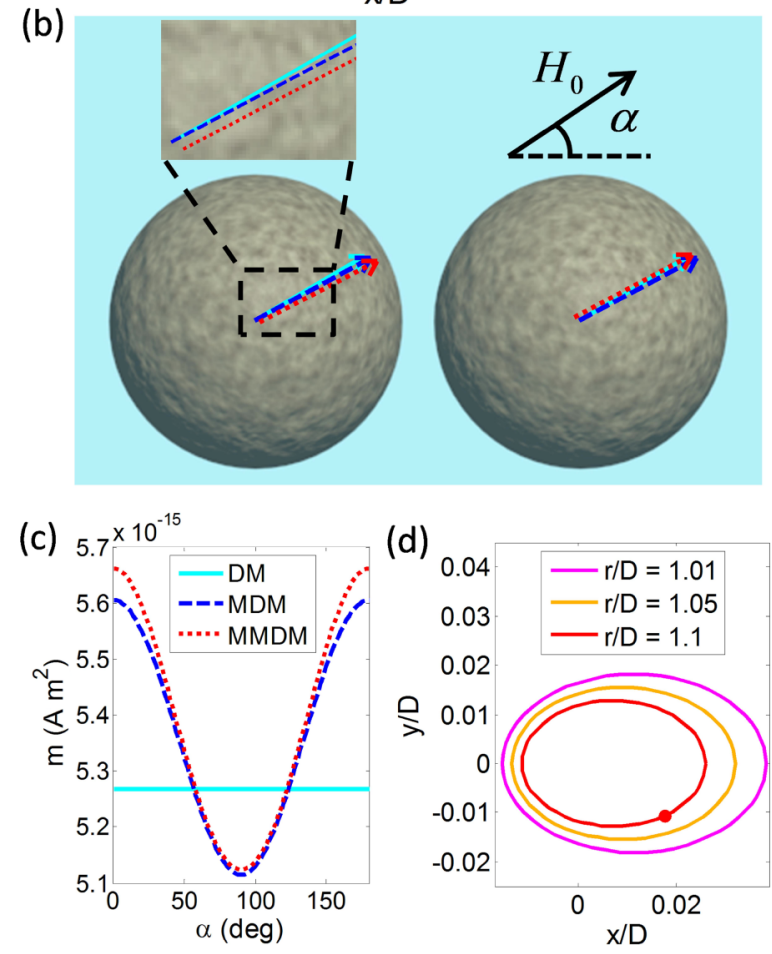

FIG. 1. (Color online) (a) The magnetic field strength distribution of a two-sphere system at the midplane $z=0$ with $\alpha=30^{\circ}$ determined by directly solving Laplace's equation for magnetostatics. For clarity, the logarithm of the magnetic field strength is plotted. The color scale represents the magnetic field strength in units of $\ln (\mathrm{A} / \mathrm{m})$. (b) The difference between the dipole moments calculated using different dipole-based models at $\alpha=30^{\circ}$. The solid arrow (cyan) denotes the DM, the dashed arrow (blue) denotes the MDM, and the dotted arrow (red) denotes the MMDM. Note that the positions of the MMDM dipole moments differ from those of the DM or MDM dipole moments as shown in the zoomed-in inset. (c) The magnitude of dipole moments calculated using different models at $r=1.1 \mathrm{D}$. (d) The trajectories of the location of the shifted dipole moments on the left particle for different center-to-center distances calculated using the MMDM. The curves from inside to outside correspond the positions of shifted dipole for $r / \mathrm{D}=1.1,1.05$, and 1.1, respectively. The red dot on the curve corresponds to the condition in (b).

to each other. Figure 1(a) shows the magnetic field strength distribution at the cross section at $z=0$ for $\alpha=30^{\circ}$, where $\alpha$ is the angle from the connector vector $\mathbf{r}$ to the applied magnetic field $\mathbf{H}_{0}$. For simplicity, we situate the two spheres on the $x$ axis and $z=0$ plane. Note that the magnetic field strength used in all of the calculations is $6 \mathrm{G}$. The spheres used in the calculations have $a=1.4 \mu \mathrm{m}$ and $\chi_{\text {eff }}=0.73$ unless otherwise noted. Because the asymmetric magnetization is the source of multipolar effects, if a dipole-based model is able to consider asymmetric magnetization, theoretically, its accuracy will approach that of the LES. In the new model, we use shifted positions of dipole moments to properly represent the effects of nonuniform magnetization. The positions of the dipole moments are calculated by taking the integral of different positions over the volume of the sphere weighted by the local magnetic field strength. In this manner, the dipole moments are no longer located at the central positions of the spheres but rather are dependent on the physical properties of the spheres and the applied magnetic field. Because the dipole moments and their positions are both different from those in the DM, $2 N$ equations must be solved simultaneously,

$$
\begin{aligned}
& \mathbf{m}_{n}=\frac{4}{3} \pi a^{3} \chi_{\text {eff }}\left(\mathbf{H}_{\mathbf{0}}+\sum_{k=1, k \neq n}^{N} \mathbf{H}_{\mathrm{dip}}\left(\mathbf{R}_{n}-\mathbf{R}_{k}\right)\right) \\
& \mathbf{R}_{n}=\frac{\int_{V} d V\left(\mathbf{r}_{n}+\mathbf{q}\right)\left|\mathbf{H}_{\mathbf{0}}+\sum_{k=1, k \neq n}^{N} \mathbf{H}_{\mathrm{dip}}\left(\mathbf{r}_{n}+\mathbf{q}-\mathbf{r}_{k}\right)\right|^{\lambda}}{\int_{V} d V\left|\mathbf{H}_{\mathbf{0}}+\sum_{k=1, k \neq n}^{N} \mathbf{H}_{\mathrm{dip}}\left(\mathbf{r}_{n}+\mathbf{q}-\mathbf{r}_{k}\right)\right|^{\lambda}} .
\end{aligned}
$$

In the above equations, $\mathbf{R}_{n}$ 's are the positions of the dipole moments, $\mathbf{q}$ is the position of the current integral volume $d V$, and $\lambda$ is the exponent of the weight factor. The Maxwell stress tensor, used in the LES method to calculate the force, depends quadratically on the magnetic field strength; thus, the exponent $\lambda$ must be greater than 2 to account for all of the multipolar effects. Using a two-sphere system, a least squared analysis of force versus $\lambda$ determined an optimized value of $\lambda=3$, which is used for the following calculations. Because of its dipolar basis and consideration of the microscopic mutual interactions, this model is referred to as the micro-mutual-dipolar model (MMDM). The dipole moments and their positions calculated using different dipole-based models are shown in Fig. 1(b). The dipole moments calculated from DM and MDM are located at the central positions of the spheres, but their directions and magnitudes are distinctly different because of the mutual interaction. The dipole moments calculated from the MMDM have directions and magnitudes that are very similar to those yielded by the MDM, but the positions are clearly offset from the central positions of the spheres because the positions are calculated using Eq. (4). This offset characterizes the asymmetric gradient of the magnetization, which is shown in Fig. 1(a); the asymmetric gradient is not considered in the MDM. The magnitude of the dipole moment is calculated for different models using an external magnetic field with different configurations, shown in Fig. 1(c). The dipole moments calculated from the MDM and MMDM have different offsets from the DM for different $\alpha$ 's. In the DM model, the dipole moment is only a function of the external field strength and does not consider mutual magnetic induction from neighboring particles. In the MMDM model, the dipole moments are shifted upwards from those calculated from the MDM, namely, they are larger in the attractive region $\left(0<\alpha \leqslant 55^{\circ}\right.$ and $\left.125^{\circ}<\alpha \leqslant 180^{\circ}\right)$ and smaller in the repulsive region $\left(55<\alpha \leqslant 125^{\circ}\right)$. The magnitude change is caused by the change in position of the dipole moments [Fig. 1(d)]. Similar to the magnitude of the dipole moments, the positions of the dipole moments also have a period of $180^{\circ}$ 
as a function of $\alpha$. The trajectories of the dipole positions are ellipses with centers closer to the neighboring particle. As the distance between particles decreases, the ellipse trajectory dilates, leading the dipole center to further lean toward the neighboring particle, as a result of the increase in asymmetric inhomogeneity of magnetization over the sphere.

The calculation of the dipole moment is the core of every dipole-based model because Eq. (1) is used for the force calculations in all of the models. Figure 2 shows the calculated force for a two-sphere system yielded by different models and the LES method. When the magnetic field is parallel to the connector vector $\left(\alpha=0^{\circ}\right)$ as shown in Fig. 2(a), the DM and MDM results both exhibit significant deviations from the LES results; the LES method is believed to perform the most-accurate force calculation because it does not employ any simplifying assumptions [17]. Nevertheless, the MMDM results agree well with the data points calculated using the LES method. Likewise, for the case described in Fig. 2(b) in which the magnetic field is perpendicular to the connector vector and $\alpha=90^{\circ}$, the MMDM provides very accurate estimations compared with the LES results. The magnetic force results at different angles when the two spheres are sufficiently close are shown in Fig. 2(c). The results yielded by different methods are clearly stratified without intersection. The two on the top are from the DM and the MDM, which are overall shifted upwards significantly from the LES results. This is a direct result of the shifted magnitude of the dipole moment shown in Fig. 1(c). The MMDM yields the best estimation with a slight deviation near the angle at which the attraction and repulsion cancel each other. This deviation is due to the nonlinearity of the multipolar effects being amplified as the attractive well dampens. Despite the slight deviation, the magnetic force calculated using the MMDM for a circular rotational magnetic (CRM) field agrees very well with that calculated using the LES as shown in Fig. 3(a).

The magnetization distributions in the spheres of a twosphere system are not symmetric because of the spheres' mutual interaction; thus, these distributions are further modified when a third sphere is added, thereby creating a three-body effect for systems with isotropic interactions $[19,20]$. The three-body effect of a three-sphere system in a CRM field can be characterized by the difference between the pair magnetic force and the effective pair magnetic force calculated for a three-sphere system. Here, the effective pair force for a three-sphere system is defined as the magnetic force on sphere 1 divided by $\sqrt{3}$, which gives the decomposed pair force from sphere 2 or 3 only [the inset at the bottom of Fig. 3(a)]. The effective pair forces for the three-sphere system calculated using the two different methods also agree well as shown in Fig. 3(a). For clarity, the MDM results are not superimposed in this figure, but one should be reminded that the pair magnetic force calculated using the MDM is slightly greater than the effective pair force for a three-sphere system calculated by using the LES (magenta squares) [17]; this offset indicates a significant deviation of the MDM results from the LES results for a CRM field. This deviation is due to the multipolar effects that exist in reality as illustrated earlier. The MMDM is capable of reproducing the LES results because it essentially uses asymmetric magnetization inside the spheres to trigger multipolar effects that are similar to the real ones. To confirm
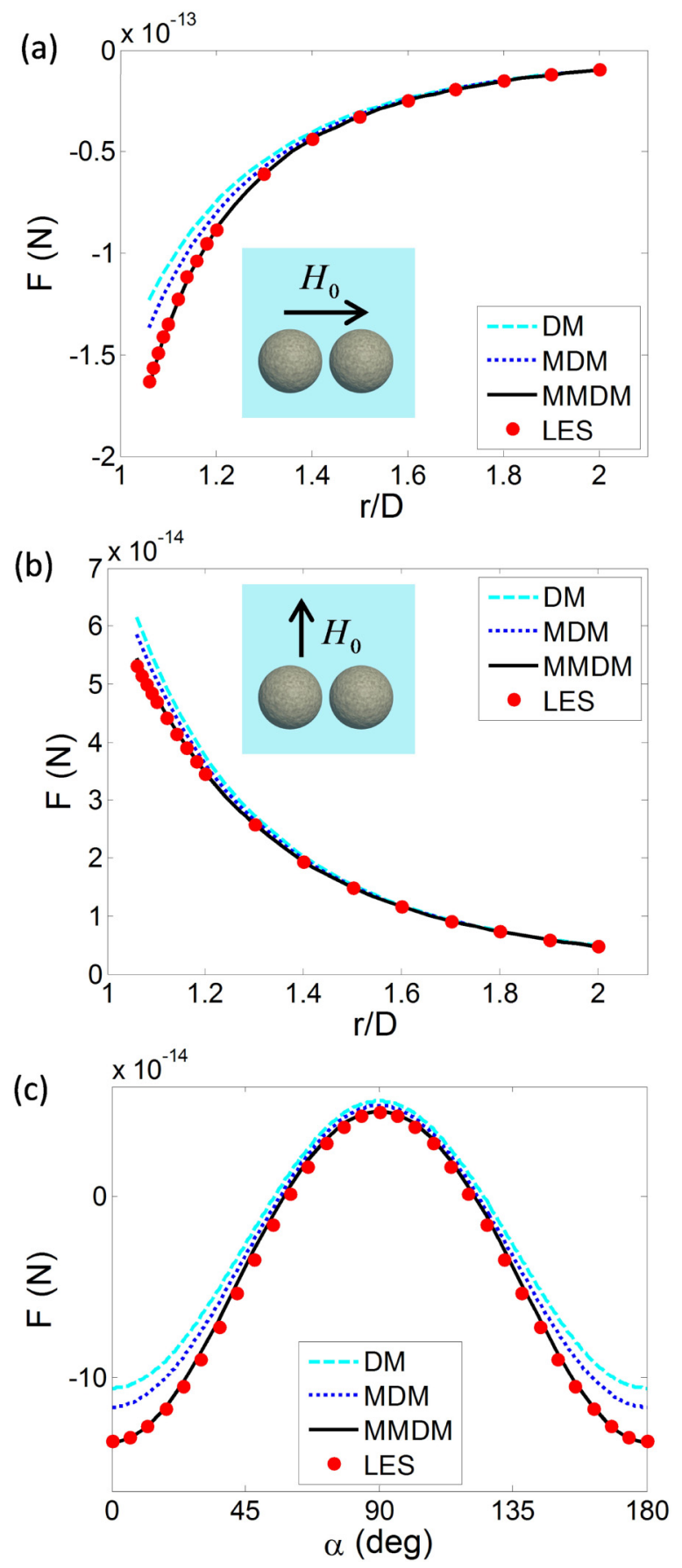

FIG. 2. (Color online) The magnetic force between two paramagnetic spheres calculated using different models (a) for different $r$ 's at $\alpha=0^{\circ}$, (b) for different $r$ 's at $\alpha=90^{\circ}$, and (c) for different $\alpha$ 's at $r=1.1 \mathrm{D}$. Note that positive values indicate repulsive forces, whereas negative values indicate attractive forces.

that the accuracy of the MMDM is not unique for spheres with $\chi_{\text {eff }}=0.73$, we further use hypothetical spheres with the same radius but $\chi_{\text {eff }}=1.88$ to calculate the same force. The inset at the top of Fig. 3(a) shows that the agreement between the LES and the MMDM results for the higher susceptibility value is as good as the agreement for the lower susceptibility value.

The failure of the MDM is mostly observed in the force calculation for spheres located along the edge of small clusters 

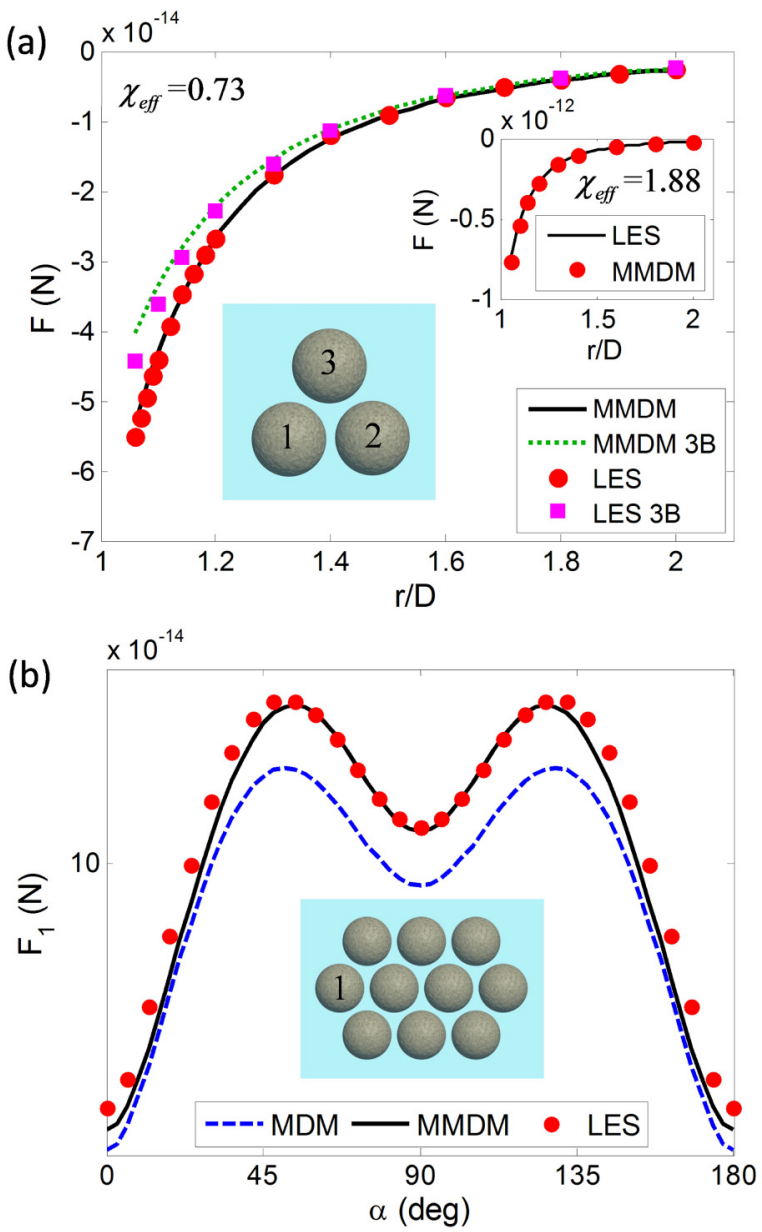

FIG. 3. (Color online) (a) The pair magnetic force and effective pair magnetic force at $r=1.1 \mathrm{D}$ for a three-sphere system in a CRM field calculated using different methods. The inset shows the pair magnetic force calculated using different methods with an increased sphere susceptibility $\chi_{\mathrm{eff}}$. (b) The magnetic force at $r=1.1 \mathrm{D}$ on sphere 1 of a ten-sphere system for different $\alpha$ 's calculated using different methods.

of spheres where the many-body effects are most significant [17]. The aforementioned three-body effect of a three-sphere system is the simplest case for the edge effect caused by many-body effects. For larger aggregates in a 2D plane, the performance of the MDM is even worse. Figure 3(b) shows the magnetic force on sphere 1 of a ten-sphere system calculated using different methods. The MDM results exhibit significant deviation from the LES results, whereas the MMDM results agree well with the LES results for all angles. However, further increasing the aggregate size may impair the accuracy of the MMDM for the edge spheres. For the edge sphere in the system that contains more than 24 spheres, the MMDM results deviate considerably from the LES results.

The definition of the MMDM does not specify the dimension of the particle system; therefore, the MMDM can also be applied to lower-dimensional spaces. In 2D space, the expressions for the dipole moments and the dipolar force must be modified. In the presence of a uniform magnetic field, when two identical disks are placed a distance $r$ from each other, the force between them can be rewritten as

$$
\begin{aligned}
\mathbf{F}= & \frac{\mu_{0}}{\pi r^{4}}\left[\left(\mathbf{m}_{1} \cdot \mathbf{r}\right) \mathbf{m}_{2}+\left(\mathbf{m}_{2} \cdot \mathbf{r}\right) \mathbf{m}_{1}+\left(\mathbf{m}_{1} \cdot \mathbf{m}_{2}\right) \mathbf{r}\right. \\
& \left.-\frac{4\left(\mathbf{m}_{1} \cdot \mathbf{r}\right)\left(\mathbf{m}_{2} \cdot \mathbf{r}\right)}{r^{2}} \mathbf{r}\right],
\end{aligned}
$$

with the same definitions of the parameters as in 3D space. The expression of the dipole moment in the MDM is modified to be

$$
\mathbf{m}_{n}=\pi a^{2} \chi_{\mathrm{eff}}\left(\mathbf{H}_{\mathbf{0}}+\sum_{k=1, k \neq n}^{N} \mathbf{H}_{\mathrm{dip}}\left(\mathbf{r}_{n}-\mathbf{r}_{k}\right)\right),
$$

where $\mathbf{H}_{\mathrm{dip}}(\mathbf{r})=\frac{1}{2 \pi} \boldsymbol{\nabla}\left(\frac{\mathbf{m} \cdot \mathbf{r}}{r^{2}}\right)=\frac{1}{2 \pi}\left(\frac{2 \mathbf{r}(\mathbf{m} \cdot \mathbf{r})}{r^{4}}-\frac{\mathbf{m}}{r^{2}}\right)$ is the dipoleinduced magnetic field and $\chi_{\text {eff }}=\frac{2 \chi}{2+\chi}$ is the effective volumetric susceptibility of the disks because the demagnetization factor in an infinitely long magnetized cylinder in the radial direction is $1 / 2$ [21]. Because Eqs. (3) and (4) are independent of the number of dimensions, they are directly used for the MMDM in 2D space. The magnetic forces between two paramagnetic disks for $\alpha=0^{\circ}$ and $\alpha=90^{\circ}$ are calculated and shown in Figs. 4(a) and 4(b), respectively. The 2D calculation yields results that are similar to those of the 3D calculation.
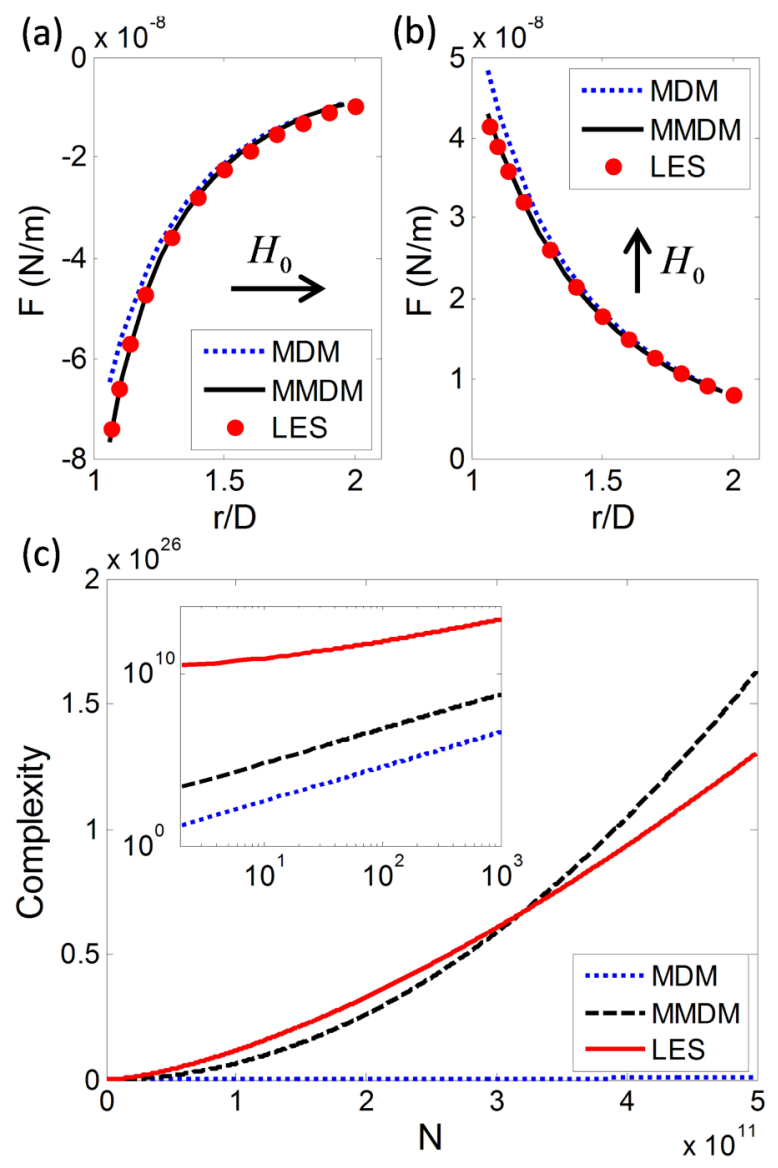

FIG. 4. (Color online) The magnetic force between two paramagnetic disks calculated using different models (a) for different $r$ 's at $\alpha=0^{\circ}$ and (b) for different $r$ 's at $\alpha=90^{\circ}$. (c) The time complexity for different sphere numbers and different methods. The inset shows the same complexity with a shorter $x$-axis interval and logarithm scales for both axes. 
The MMDM and LES results agree well, whereas the MDM results deviate considerably. Note that the calculated force is essentially over a unit axial length of a cylinder with a circular cross section and therefore has a unit of $\mathrm{N} / \mathrm{m}$. In practice, the MMDM can be used to calculate the force between long cylinders with a circular cross section as a complement to the calculation for long needles with square cross sections [22,23].

In addition to accuracy, another important factor that determines the effectiveness of a numerical method is its time complexity. The time complexity is expressed as a function of the input size, namely, the number of particles $N$. Here, we use the $3 \mathrm{D}$ case as an example, and the complexity for the 2D case can be derived similarly. Because dipole-based models must scan over all the possible combinations of particle pairs to calculate the force, the MDM and MMDM have a complexity that is on the order of $N^{2}$. The LES method does not have to calculate the pairwise force, but it has to propagate the infinite boundary conditions in 3D space toward the center. For an aggregate in a 2D plane, the complexity is on the order of $N^{3 / 2}$. Figure 4(b) shows the time complexity of different methods. Although the complexity of the LES method grows more slowly with increased $N$, it has a much higher starting point as shown in the inset of Fig. 4(b). Thus, the computational time of the LES method is usually more than 5 orders of magnitude greater than that of the dipole-based models when the system consists of less than 1000 particles. The LES method is faster than the MMDM only when $N>3 \times 10^{11}$ as shown in Fig. 4(b). In computer simulations of particle systems, because of the applied periodic boundary conditions, the number of particles is usually no greater than $10^{6}$. This fact restricts the application of the LES method and makes the MMDM both accurate and efficient for magnetic force calculations.

The MMDM has been demonstrated to be able to accurately and quickly calculate the magnetic force between paramagnetic spheres or disks for small aggregates. The extra accuracy compared with the MDM originates from the offset positions of the dipole moments, which represent the asymmetry of magnetization over the material. For small aggregates, the MMDM is able to accurately calculate the forces on exterior particles which have large asymmetric inhomogeneity of magnetization. For interior particles, the inhomogeneity decreases due to the surrounding particles that are more symmetrically situated. The force on the interior particles can be therefore accurately approximated by superimposing the pair LES force results. For large aggregates, the performance of the MMDM is impaired for exterior particles. Moreover, large aggregates have more interior particles, whose configurations are more important for determining different order functions. Thus MMDM is an outstanding model for calculating magnetic forces for small aggregates and has potential applications for dynamical simulations without periodic boundary conditions.

We gratefully thank S. Kang and F. Zhang for insightful discussions. We acknowledge the National Science Foundation (Grant No. CBET-0955003) and Welch Foundation (Grant No. C-1755) for financial support.
[1] H. Zhang and M. Widom, Phys. Rev. E 51, 2099 (1995).

[2] D. Du, D. Li, M. Thakur, and S. Biswal, Soft Matter 9, 6867 (2013).

[3] N. Osterman, I. Poberaj, J. Dobnikar, D. Frenkel, P. Ziherl, and D. Babić, Phys. Rev. Lett. 103, 228301 (2009).

[4] J. E. Martin, R. A. Anderson, and R. L. Williamson, J. Chem. Phys. 118, 1557 (2003).

[5] S. Melle and J. E. Martin, J. Chem. Phys. 118, 9875 (2003).

[6] S. L. Biswal and A. P. Gast, Phys. Rev. E 69, 041406 (2004).

[7] U. Jeong, X. W. Teng, Y. Wang, H. Yang, and Y. N. Xia, Adv. Mater. 19, 33 (2007).

[8] Z. Ye and M. Sitti, Lab Chip 14, 2177 (2014).

[9] R. Dreyfus, J. Baudry, M. L. Roper, M. Fermigier, H. A. Stone, and J. Bibette, Nature (London) 437, 862 (2005).

[10] P. Tierno, O. Guell, F. Sagues, R. Golestanian, and I. Pagonabarraga, Phys. Rev. E 81, 011402 (2010).

[11] D. C. Li, J. Rogers, and S. L. Biswal, Langmuir 25, 8944 (2009).

[12] R. Dreyfus, D. Lacoste, J. Bibette, and J. Baudry, Eur. Phys. J. E 28, 113 (2009).
[13] D. Griffiths, Introduction to Electrodynamics, 3rd ed. (Prentice Hall, Upper Saddle River, NJ, 1999).

[14] E. E. Keaveny and M. R. Maxey, J. Comput. Phys. 227, 9554 (2008).

[15] J. D. Jackson, Classical Electrodynamics, 3rd ed. (Wiley, New York, 1998).

[16] H. J. H. Clercx and G. Bossis, Phys. Rev. E 48, 2721 (1993).

[17] D. Du, F. Toffoletto, and S. L. Biswal, Phys. Rev. E 89, 043306 (2014).

[18] E. O. Steinborn and E. Filter, Theor. Chem. Acc. 38, 261 (1975).

[19] J. Dobnikar, M. Brunner, H.-H. von Grünberg, and C. Bechinger, Phys. Rev. E 69, 031402 (2004).

[20] C. Russ, M. Brunner, C. Bechinger, and H. H. von Grünberg, Europhys. Lett. 69, 468 (2005).

[21] M. Sato and Y. Ishii, J. Appl. Phys. 66, 983 (1989).

[22] V. A. Ignatchenko, H. Kronmüller, and M. Grönefeld, J. Magn. Magn. Mater. 89, 229 (1990).

[23] G. Rowlands, J. Magn. Magn. Mater. 118, 307 (1993). 